\title{
Frontal cortical synaptophysin in Lewy body diseases: relation to Alzheimer's disease and dementia
}

\author{
L A Hansen, S E Daniel, G K Wilcock, S Love
}

Departments of Pathology and

Neurosciences, University of

California, San Diego, La Jolla, California, USA

L A Hansen

Parkinson's Disease Society Brain Bank, London, UK

S E Daniel

Department of Care of the Elderly

G K Wilcock

Department of Neuropathology, University of Bristol, Frenchay Hospital, Bristol, UK

S Love

Correspondence to: Dr L A Hansen, Department of Pathology, University of California, San Diego, 9500 Gilman Drive, La Jolla, California 92093-0624, USA. Telephone 001619 534 6212; fax: 001619534 6232; email:

lahansen@UCSD.edu

Received 27 August 1997 and in revised form 7 October 1997

Accepted 16 October 1997

\begin{abstract}
Objectives-Dementia in Alzheimer's disease correlates closely with loss of neocortical synapses. Similar synaptic loss has been shown in patients whose Alzheimer's disease is also associated with neocortical and brain stem Lewy bodies. The aim was to determine if dementia in Lewy body disease was associated with diminished concentrations of midfrontal cortex synaptophysin.

Methods-An immunobinding assay was used to measure synaptophysin in postmortem samples of midfrontal cortex from 89 patients with Alzheimer's disease (ages 59-100, mean 79), 22 with combined Lewy body disease and Alzheimer's disease (ages 69-103, mean 79), 15 demented patients with "pure" Lewy body disease (ages 57-80, mean 74), nine with neocortical and brain stem Lewy bodies who had Parkinson's disease but were not demented (ages 68-85, mean 79), and 20 neurologically normal controls (ages 58-89, mean 75). The diagnosis was confirmed in all cases by detailed neuropathological examination of the contralateral hemibrain. Seven of the patients in the pure Lewy body disease with dementia group had initially presented with parkinsonism and eight with dementia.
\end{abstract}

Results-Synaptophysin concentrations (arbitrary units (AU)/ $/ \mu \mathrm{g}$ ) in patients with Alzheimer's disease (mean 79 (SD 28)) or combined Lewy body disease and Alzheimer's disease (mean 83 (SD 33)) were significantly lower than in controls (mean 115 (SD 29)) ( $=0.002)$. Synaptophysin concentrations in demented patients with pure Lewy body disease (mean 106 SD 39) and patients with Lewy body disease who were not demented (mean 101 (SD 18)) did not differ significantly from control values or from each other.

Conclusion-Loss of midfrontal cortex synapses probably contributes to dementia in Lewy body disease when Alzheimer's disease is also present but not to the dementia of pure Lewy body disease.

(F Neurol Neurosurg Psychiatry 1998;64:653-656)

Keywords: Lewy body disease; dementia; synaptophysin

Lewy body diseases include idiopathic Parkinson's disease and dementia with Lewy bodies (DLB). Parkinson's disease is primarily a movement disorder, but up to $40 \%$ of patients with Parkinson's disease eventually become demented. ${ }^{1}$ DLB is a more recently recognised clinicopathological entity which accounts for perhaps $20 \%$ of dementia in elderly people. ${ }^{2}$ It is typically associated with mild parkinsonism, and usually resting tremor is absent. Both Parkinson's disease and DLB show neuronal loss and gliosis in the substantia nigra, locus ceruleus, and the nucleus basalis of Meynert, accompanied by single or multiple Lewy bodies in surviving neurons, and at least some neocortical Lewy bodies can be found in both conditions. The neuropathological abnormalities associated with dementia in Parkinson's disease and DLB are variable and complex. About a third of demented patients with Parkinson's disease have concomitant Alzheimer's disease at necropsy, another $10 \%$ have widespread neocortical Lewy bodies, and most of the remainder show only the typical pathology of Parkinson's disease. ${ }^{1}$ Most DLB brains have more Alzheimer's disease pathology than age matched controls, but far less than is encountered in typical Alzheimer's disease. ${ }^{3}$ These cases of mixed Lewy body disease and Alzheimer's disease have also been termed Lewy body variants of Alzheimer's disease (LBV). ${ }^{4}$ Almost $30 \%$ of DLB cases, however, are free of changes associated with Alzheimer's disease. Patients with such pure Lewy body disease are less demented than those with both Lewy bodies and Alzheimer's disease lesions. ${ }^{56}$ Dementia in Alzheimer's disease correlates closely with loss of neocortical synapses, ${ }^{7}$ and similar synaptic loss has been found in patients with combined Alzheimer's disease and Lewy body pathology. ${ }^{6}$ In this study, we sought to determine if dementia across the range of Lewy body diseases, including both Parkinson's disease and DLB, is associated with loss of midfrontal cortex synaptophysin, as is the case in Alzheimer's disease.

\section{Materials and methods}

SUBJECTS

We evaluated demented and non-demented patients with Lewy body disease to determine if synaptophysin loss could explain cognitive dysfunction. To control for the confounding influence of concurrent Alzheimer's disease pathology, we separated cases of DLB into two groups: those with and those without concomitant lesions of Alzheimer's disease. Finally, we contrasted the results in these groups with those obtained from a cohort of patients with pure Alzheimer's disease, and results from age matched non-demented controls. 
Mean (SD) concentrations of synaptophysin

\begin{tabular}{|c|c|c|c|c|}
\hline & $n$ & Mean age & $\begin{array}{l}\text { Mean PM } \\
\text { delay }(h)\end{array}$ & $\begin{array}{l}\text { Mean synaptophysin } \\
(A U / \mu g)\end{array}$ \\
\hline LBD without dementia & 9 & $79(5)$ & $15(14)$ & $101(18)$ \\
\hline LBD with dementia & 15 & $74(7)$ & $26(17)$ & $106(39)$ \\
\hline Mixed LBD + AD & 22 & $79(8)$ & $11(10)$ & $83(33)$ \\
\hline $\mathrm{AD}$ & 89 & $79(9)$ & NA & 79 (28) \\
\hline Controls & 20 & $75(8)$ & $42(35)$ & $115(29)$ \\
\hline
\end{tabular}

LBD = Lewy body disease AD = Alzheimer's disease NA = not available, but $<24$ hours; $\mathrm{PM}=$ postmortem.

The non-demented Lewy body disease group consisted of nine patients with clinically diagnosed idiopathic Parkinson's disease (age range $68-85$, mean 74 ), whose brains showed only typical Parkinson's disease pathology, including a few neocortical Lewy bodies in every instance, without Alzheimer's disease changes. The DLB group included 37 patients, both those whose initial and primary clinical diagnosis was dementia, and patients with Parkinson's disease who developed dementia before death. These 37 DLB specimens were separated into those with and those without concomitant Alzheimer's disease; a neuropathological diagnosis of Alzheimer's disease was assigned if a brain had enough senile plaques to meet both National Institute on Aging criteria for Alzheimer's disease ${ }^{8}$ and criteria from the Consortium to Establish a Registry for Alzheimer Disease for "definite", or "probable" Alzheimer's disease. . There were 22 such mixed Lewy body disease and Alzheimer's disease brains. All 22 of these patients (age range 69-103, mean 79) had presented with dementia, not Parkinson's disease. The remaining 15 DLB cases (age range 57-80, mean 74) did not meet the above described neuropathological criteria for Alzheimer's disease. Seven of these patients had presented initially with Parkinson's disease, and eight with dementia. The Alzheimer's disease comparison group consisted of 89 patients with pure Alzheimer's disease (age range 59-100, mean 79). Finally, we also analysed synaptophysin concentrations from 20 age matched non-demented controls (age range 58-89, mean 75).

NEUROPATHOLOGICAL EVALUATION

At necropsy, the brains were divided midsagittally, and the right hemibrain was frozen at $-70^{\circ} \mathrm{C}$ for neurochemical analysis, and the left was fixed in formalin for 10 days to three weeks before dissection. Standard neuropathological evaluation was performed on sections of frontal, temporal, and parietal lobes, hippocampus, entorhinal cortex, basal ganglia, substantia innominata, mesencephalon, and rostral pons. Neuronal loss, gliosis, and Lewy bodies were identified in the pigmented nuclei of the brain stem and nucleus basalis of Meynert in haematoxylin and eosin stained sections. All brains with Lewy bodies in the brain stem and nucleus basalis of Meynert included at least some neocortical Lewy bodies, predominately distributed in the limbic and entorhinal cortices and demonstrable in sections stained with haematoxylin and eosin and immunohistochemically for ubiquitin. The extent of
Alzheimer's disease pathology was evaluated using either thioflavin-S preparations viewed under ultraviolet illumination, or with modified Bielschowsky silver impregnation for plaques and tangles.

\section{MIDFRONTAL CORTEX SYNAPTOPHYSIN}

QUANTIFICATION

Specimens from the frozen right hemibrain midfrontal cortex were analysed with a dot immunobinding assay for quantification of synaptophysin-like immunoreactivity. Postmortem intervals before freezing of the brain tissue varied considerably, from two to 103 hours, but we have shown this method of synaptophysin measurement to be stable over prolonged postmortem intervals, ${ }^{10}$ and the controls had both the longest average postmortem delay interval (42 hours) and the highest mean synaptophysin concentrations (115 arbitrary units (AU)/ $/ \mu \mathrm{g})$. Determination of neocortical synaptophysin was undertaken by homogenising $0.1 \mathrm{~g}$ frozen brain tissue in $1 \mathrm{ml}$ cold homogenising buffer with a Dounce glass/glass tissue grinder. A low speed spin (5000 $g \times 10$ minutes) was performed and the supernatant separated into particulate and cytosolic fractions by ultracentrifugation $(356000 g \times 1 \mathrm{~h}$, $\left.4^{\circ} \mathrm{C}\right)$. After resuspending pellet with buffer, 2 $\mu \mathrm{g}$ particulate fraction was blotted on to nitrocellulose membrane using a dot-blot manifold. A serial dilution of aliquoted control sample was included as a normalisation standard. Blots were blocked using phosphate buffered saline (PBS) and $0.1 \%$ Tween-20 for two hours. They were then incubated in primary antibody (antisynaptophysin, BoehringerMannheim Biochemical, Indianapolis, IN, USA, mouse monoclonal, $0.1 \mu \mathrm{g} / \mathrm{ml}$ in PBSBSA) for 12-18 hours, followed by rabbit antimouse IgG $\left(0.85 \mu \mathrm{g} / \mathrm{ml}\right.$ in PBS-BSA), and ${ }^{125} \mathrm{I}-$ protein A $(0.1 \mu \mathrm{Ci} / \mathrm{ml}$ in PBS-BSA) for two hours each. After washing in PBS-Tween, an autoradiogram was produced on a phosphor imaging screen and quantified on a Molecular Dynamics phosphorImager SE using the ImageQuant Software (Molecular Dynamics, Sunnyvale, CA, USA) . Results are expressed as arbitrary units (AU)/ $\mu \mathrm{g}$ blotted protein after normalising against the standard curve.

\section{Results}

The table shows the result of midfrontal cortex synaptophysin assay in each group. Unpaired two tailed $t$ tests were used for the statistical analyses of the differences in midfrontal cortex synaptophysin concentrations. p Values $<0.01$ were regarded as statistically significant. Midfrontal cortex synaptophysin concentrations in patients with Alzheimer's disease, or combined Lewy body disease and Alzheimer's disease were significantly lower than in controls $(\mathrm{p}=0.001$ and $\mathrm{p}=0.002$, respectively). Further statistical analysis by one way analysis of variance (ANOVA) showed that mean synaptophysin values for the four groups differed significantly $(F(3,62)=3.62, \mathrm{p}<0.02)$. Post hoc comparisons by the least squares method showed that the combined Lewy body disease and Alzheimer's disease groups had a 
significantly lower synaptophysin value than controls $(p<0.003)$. Synaptophysin concentrations in demented patients with only Lewy body disease and patients with Lewy body disease who were not demented did not differ significantly from control values or from one another.

\section{Discussion}

The results indicate that dementia in Lewy body disease is not associated with loss of midfrontal synaptophysin unless there is concomitant Alzheimer's disease pathology. Synaptic loss in the midfrontal cortex cannot, therefore, serve as a unitary explanation for dementia across the clinical range of Lewy body diseases. These findings confirm those of a previous investigation into synaptic loss in the frontal cortex in DLB, which had employed far fewer cases, and they extend the finding to include patients who present initially with Parkinson's disease and subsequently become demented. Samuel et $a l^{6}$ reported that brains showing mixed Lewy body disease and Alzheimer's disease (LBV in their nomenclature) had significantly lower midfrontal cortex synaptophysin concentrations than had specimens from demented patients with only Lewy body disease (referred to by the authors as diffuse Lewy body disease, DLBD). Midfrontal cortex synapse loss in LBV was similar to that seen in Alzheimer's disease, whereas synaptophysin concentrations in DLBD did not differ from controls.

Our results imply that dementia in Lewy body disease is due to neuropathological changes other than synaptic loss in the midfrontal cortex. Modified Braak stages of Alzheimer's disease neurofibrillary pathology ${ }^{11}$ and neocortical neuritic plaque counts correlate with dementia in $\mathrm{DLB},{ }^{6}$ and increasing Braak stages are also associated with worsening cognition in combined Parkinson's disease and Alzheimer's disease. ${ }^{12}$ It is not surprising that progressively increasing burdens of Alzheimer's disease pathology correlate with dementia, when such lesions are present in many patients with Lewy body disease. Neuropathological overlap of Alzheimer's disease and Lewy body disease is well established, both neuropathologically and genetically. Lewy bodies have been found in some cases of familial Alzheimer's disease due to a codon 717 mutation in the amyloid precursor protein gene. ${ }^{13}$ Cortical Lewy bodies have also been reported in patients with Down's syndrome, who invariably develop Alzheimer's disease if they live long enough. ${ }^{14}$ Finally, brains showing Lewy body disease with concomitant Alzheimer's disease plaques and entorhinal cortex neurofibrillary tangles, whether termed Alzheimer's disease plus Parkinson's disease ${ }^{15}$ or LBV, ${ }^{16}$ have increased frequencies of the apolipoprotein E4 allele, similar to those encountered in Alzheimer's disease. In patients with pure Lewy body disease, whether or not associated with dementia, the frequency of the apolipoprotein E4 allele is similar to that in the normal population. ${ }^{17}$ Concomitant Alzheimer's disease cannot, however, explain impaired cognition in neuropathologically pure DLB or Parkinson's disease. Neuropsychological comparisons of DLBD and LBV show significantly less dementia in patients with DLBD, ${ }^{56}$ but average mini mental state examination scores of 17 and Blessed scores of 13 among the patients with DLBD clearly establish the presence of disabling mental impairment without Alzheimer's disease changes.

Neocortical choline acetyltransferase (ChAT) is strikingly diminished in DLB, far more so than in Alzheimer's disease, which nevertheless shows significant reductions compared with controls. ${ }^{4}$ In patients with Parkinson's disease, ChAT reductions in the temporal neocortex correlate with the degree of mental impairment assessed by a test of memory and information, and with neuronal counts in the nucleus of Meynert. ${ }^{18}$ Depletion of ChAT in the midfrontal cortex has also been shown to correlate with severity of dementia in DLB, ${ }^{19}$ and similar results have been reported in the pathologically identical condition termed senile dementia of the Lewy body type. ${ }^{20}$

Neocortical Lewy body counts correlate with dementia in DLB, ${ }^{6} \mathrm{LBV}^{21}$ and DLBD. ${ }^{22}$ As neocortical Lewy bodies are generally, although not invariably, more frequent in DLB than Parkinson's disease, their appearance and proliferation may constitute an additional explanation for the emergence of dementia in patients with Parkinson's disease without concomitant Alzheimer's disease pathology.

Damage to dopaminergic frontostriatal neurochemical mechanisms has been implicated as a possible neuropathological substrate for dementia in DLB and Parkinson's disease with dementia. A recent analysis of concentrations of homovanillic acid in CSF found significantly lower concentrations in LBV than in Alzheimer's disease. ${ }^{23}$ The authors concluded that Lewy bodies in the mesolimbic and mesocortical regions may be partly responsible for the reduced dopamine metabolism and turnover reflected in low CSF concentrations of homovanillic acid, and that this may be related to the frequency of psychotic symptoms in this disorder. Brains from patients with Parkinson's disease also show much lower amounts of dopamine and its metabolites in the hippocampus, and entorhinal, cingulate, and frontal cortex than do controls, ${ }^{24}$ and such dopaminergic deficiency may contribute to mental impairment in Parkinson's disease. Depletion of other subcortical projecting systems in Lewy body diseases could also impair cognition. In particular, decreased concentrations of serotonin and noradrenaline, secondary to neuronal loss in the locus ceruleus and raphe nuclei, may contribute to dementia in DLB and Parkinson's disease.

Our finding that neocortical synaptic loss does not contribute to dementia in DLB or Parkinson's disease, unless there is concomitant Alzheimer's disease pathology, applies only to the midfrontal cortex. Although neocortical Lewy bodies do occur in the frontal lobe in DLB and Parkinson's disease, they are more numerous in the limbic cortex, and so synaptic loss in the entorhinal cortex, cingulate gyrus, or 
insular may yet prove to impair cognition in Lewy body diseases. The data available at present, however, imply that concomitant Alzheimer's disease pathology in the form of neuritic plaques, higher Braak stages, and midfrontal cortex synaptic loss worsens the moderately severe dementia encountered in neuropathologically pure Lewy body diseases. In the absence of Alzheimer's disease changes, dementia in Lewy body diseases seems to be associated with a complex combination of neocortical Lewy body formation, loss of neocortical ChAT and dopamine, and progressive neurodegeneration in multiple cortically projecting brain stem nuclei.

1 Hughes AJ, Daniel SE, Blankson S, et al. A clinicopathologic study of 100 cases of Parkinson's disease. Arch Neurol 1993;50:140-53.

2 McKeith IG, Galasko D, Kosaka K, et al. Consensus guidelines for the clinical and pathological diagnosis of dementia with Lewy bodies (DLB): report of the consortium on DLB international workshop. Neurology 1996;47:1113-24.

3 Hansen, LA, Samuel W. Criteria for Alzheimer disease and the nosology of dementia with Lewy bodies. Neurology the nosology of

4 Hansen L, Salmon D, Galasko D, et al. The Lewy body variant of Alzheimer's disease: a clinical and pathological ant of Alzheimer's disease:

5 Salmon DP, Galasko D, Hansen LA, et al. Neuropsychological deficits associated with diffuse Lewy body disease. Brain Cogn 1996;31:148-65.

6 Samuel W, Alford M, Hofstetter R, et al. Dementia with Lewy bodies versus pure Alzheimer disease: differences in cognition, neuropathology, cholinergic dysfunction, and synapse density. F Neuropathol Exp Neurol 1997;56:41-50.

7 Terry RD, Masliah E, Salmon D, et al. Physical basis of cognitive alterations in Alzheimer disease: synapse loss is the major correlate of cognitive impairment. Ann Neurol 1991; 30:572-80.

8 Khachaturian ZS. Diagnosis of Alzheimer's disease. Arch Neurol 1985;42:1097-105.

9 Mirra SS, Hart MN, Terry RD. Making the diagnosis of Alzheimer's disease. Arch Pathol Lab Med 1993;117:13244. 10 Alford MF, Masliah E, Hansen LA, et al. A simple synaptophysin-like immunoreactivity in human brain. $\mathcal{f}$ Histochem Cytochem 1994;42:283-7.

11 Braak H, Braak E. Neuropathological staging of Alzheimerrelated changes. Acta Neuropathol 1991;82:239-59.

12 Banshor C, Braak H, Fischer P, et al. Neuropathological staging of Alzheimer lesions and intellectual status in Alzheimer's and Parkinson's disease patients. Neurosci Lett 1993;162:179-82.

13 Lantos PL, Ovenstone IMK, Johnson J, et al. Lewy bodies in the brain of two members of a family with the 171 (Val to Ile) mutation of the amyloid precursor protein gene. Neurosci Lett 1994;172:77-9.

14 Bodhireddy S, Dickson DW, Mattiace L, et al. A case of Down's syndrome with diffuse Lewy body disease and Alzheimer's disease. Neurology 1994;44:159-61.

15 Gearing M, Schneider JA, Rebec GW, et al. Alzheimer's disease with and without coexisting Parkinson's disease changes: apolipoprotein E genotype and neuropathologic correlates. Neurology 1995;45:1985-90.

16 Galasko D, Saitoh T, Xia Y, et al. The apolipoprotein E allele $\varepsilon 4$ is overrepresented in patients with the Lewy body variant of Alzheimer's disease. Neurology 1994;44:1950.

17 Nalbantoglu J, Gilfix BM, Bertrand P, et al. Predictive value of apolipoprotein $\mathrm{E}$ genotyping in Alzheimer's disease: results of an autopsy series and an analysis of several comresults of an autopsy series and an analysis of
bined studies. Ann Neurol 1994;36:889-95.

18 Perry EK, Curtis M, Dick DJ, et al. Cholinergic correlates of cognitive impairment in Parkinson's disease: comparisons with Alzheimer's disease. $f$ Neurol Neurosurg Psychiatry 1985;48:413-21.

19 Perry EK, Marshall E, Perry RH, et al. Cholinergic and dopaminergic activities in senile dementia of Lewy body type. Alzheimer Dis Assoc Disord 1990;4:87-95.

20 Perry EK, McKeith I, Thompson P, et al. Topography, extent and clinical relevance of neurochemical deficits in dementia of Lewy body type, Parkinson's disease, and Alzheimer's disease. Ann NY Acad Sci 1991;640:197-202.

21 Samuel W, Galasko D, Masliah E, et al. Neocortical Lewy body counts correlate with dementia in the Lewy body variant of Alzheimer's disease. I Neuropathol Exp Neurol 1996;55:44-52.

22 Lennox G, Lowe J, Landon M, et al. Diffuse Lewy body disease: correlative neuropathology using anti-ubiquitin immunocytochemistry. I Neurol Neurosurg Psychiatry 1989; 52:1236-47.

23 Weiner MF, Risser RC, Cullum CM. et al. Alzheimer's disease and its Lewy body variant: a clinical analysis of postmortem verified cases. Am f Psychiatry 1996;153: 1269-73.

24 Scatton B, Rouquier L, Javoy-Agid F, et al. Dopamine deficieny in the cerebral cortex in Parkinson disease. Neurology 1982;23:1039-40. 